\title{
Time trends in mortality rates in type 1 diabetes from 2002 to 2011
}

\author{
Marit E. Jørgensen • Thomas P. Almdal • \\ Bendix Carstensen
}

Received: 25 June 2013 / Accepted: 26 July 2013 /Published online: 16 August 2013

(C) Springer-Verlag Berlin Heidelberg 2013

\begin{abstract}
Aims/hypothesis The aim of this study was to estimate absolute and relative mortality rates in patients with type 1 diabetes at the Steno Diabetes Centre relative to the general Danish background population.

Methods Patients with type 1 diabetes $(n=4,821)$ were followed from 1 January 2002 until 1 January 2011, with death from any cause as the main outcome. Poisson regression was used to model mortality rates by age, diabetes duration and calendar time, according to sex.

Results In the period 2002-2010, a total of 673 deaths (402 men, 271 women) occurred in the study population during 33,847 years of follow-up of type 1 diabetes. The predominant cause of death in patients with type 1 diabetes was cardiovascular disease. Mortality rates were highest among those with the lowest age at onset, particularly men. The mortality rate in the diabetic population decreased over that time period by $6.6 \%$ and $4.8 \%$ per year in men and women, respectively; this was substantially greater than the decrease in mortality rates in the background population. The decline in standard mortality rate was $4.3 \%$ per year in men and $2.6 \%$ per year in women. Patients who did not develop nephropathy had only slightly elevated mortality rates compared with the background population.

Conclusions/interpretation Despite advances in care, mortality rates in the past decade continue to be greater in patients with type 1 diabetes than in those without diabetes; however, the mortality rate in patients decreased over the study period faster than that of the background population. Nephropathy seems to be the main driver of the excess mortality.
\end{abstract}

M. E. Jørgensen $(\bowtie) \cdot$ T. P. Almdal • B. Carstensen

Steno Diabetes Centre, Niels Steensensvej 2,

DK-2820 Gentofte, Denmark

e-mail:MAEJ@steno.dk
Keywords Complications $\cdot$ Epidemiology $\cdot$ Mortality rate Nephropathy · Type 1 diabetes

\author{
Abbreviations \\ EPR Electronic patient record \\ SMR Standardised mortality rate
}

\section{Introduction}

Mortality rates in type 1 diabetes are higher than in the non-diabetic population [1]. Most of the excess mortality in early-onset type 1 diabetes is due to long-term complications, particularly nephropathy [2, 3], but knowledge about mortality rates in late-onset and elderly type 1 diabetes is scarce.

It is not clear to what extent recent advances in diabetes care have reduced the excess mortality in type 1 diabetes. To our knowledge, no information on mortality rates in patients with type 1 diabetes with follow-up since 2007 has been published [4].

The aim of this study was to estimate absolute and relative mortality rates for a well-defined population with type 1 diabetes in Denmark, as well as trends in mortality rates in the period from 1 January 2002 to 31 December 2010 for patients with and without nephropathy including the effect of duration of diabetes.

\section{Methods}

Register data All Danish residents have a unique personal identification number recorded in the Danish Civil Registration System. Patients with type 1 diabetes in Denmark are exclusively managed in hospital outpatient clinics. The Steno Diabetes Centre is a specialised diabetes hospital, but works as 
an integrated part of the public healthcare system. According to information from the National Quality Assurance Database for Diabetes, the type 1 diabetes population of the Steno Diabetes Centre constitutes a representative sub-sample of the total Danish adult type 1 diabetes population, and quality standards are comparable to other type 1 diabetes units.

Information on diabetes and complications In the Steno electronic patient records (EPRs), type 1 diabetes is clinically diagnosed on the basis of phenotype: age below 30 years and treatment with insulin at diagnosis. Patients above 30 years of age at diagnosis or who were initially using oral hypoglycaemic drugs before treatment with insulin are classified as type 1 diabetic patients if there is an absolute need for insulin to control blood glucose in combination with low C-peptide values or glutamic acid decarboxylase (GAD)65 antibody positivity. Diabetic nephropathy is measured annually and defined as a urinary albumin/creatinine ratio $\geq 3.5 \mathrm{mg} / \mathrm{mmol}(30 \mathrm{mg} / \mathrm{g})$ in two of three consecutive sterile urine specimens. Information on date of diagnosis and other complications is available in the EPR. According to information from the National Quality Assurance Database for Diabetes, annual measurements of the relevant process indicators (nephropathy, retinopathy, neuropathy) are performed in $96-99 \%$ of patients [5].

Mortality rates and causes of death in type 1 diabetes From Statistics Denmark's databank, we obtained data on the population size from 1 January 2002 to 31 December 2010 by sex and age, as well as the number and causes of deaths in the Danish population.

The type 1 diabetes data were linked to mortality data from the Civil Registration System by the personal identification number, and mortality rates were calculated.

Data analysis Patients were followed from the start of the EPR system (1 January 2002) until the last update of the EPR with the Civil Registration System (31 December 2010) or death. Patient follow-up was classified by presence of nephropathy or not (a time-dependent variable). Patient followup (risk time and deaths) was split into 3-month intervals, each recording the current age, date, diabetes duration and complication status. For each interval, we attached the corresponding population mortality rate (now in 1-year age and calendar time intervals) and computed the expected number of deaths.

The time-split data were analysed with a Poisson model, using $\log _{\mathrm{e}}$ (person-time) or $\log _{\mathrm{e}}$ (expected deaths) as offset for analysis of mortality rates and standardised mortality rates (SMRs). Mortality rates and SMRs were analysed with smooth terms of current age, duration of diabetes and nephropathy status, with a simple one-parameter (product) interaction between age and diabetes duration. Since the focus was on effects of clinical management over time, we included a linear complication-by-calendar interaction. All analyses and graphs were generated with the statistical program ' $R$ ' version 3.0.1 (www.R-project.org) [6]. A complete account of all statistical analyses is available at http://bendixcarstensen. com/SDC/EPJmort/Mort.pdf.

Ethics approval This is not required for register studies in Denmark. Access and use of the described data are approved by the Danish Data Protection Agency (j-No: 2007-58-0015).

\section{Results}

The population comprised 5,363 patients with type 1 diabetes. Patients without a date of diagnosis were excluded, leaving 4,821 for analysis. A description of the total population is given in Table 1.

Total mortality by age, diabetes duration and nephropathy In the period 2002-2010, a total of 673 deaths (402 men, 271 women) occurred in the population during 33,847 years of follow-up. Cardiovascular disease was the predominant cause of death, followed by cancer (Table 1). The mortality rate among patients with and without nephropathy by age, age at onset and sex is shown in Fig. 1a, b. The mortality rate

Table 1 Patient characteristics at study entry and causes of death during follow-up

\begin{tabular}{lll}
\hline & Men & Women \\
\hline Baseline characteristics & & \\
Number & $2,614(54)$ & $2,207(46)$ \\
Median age (years) & $44(33-56)$ & $43(32-57)$ \\
Median diabetes duration (years) & $18(8 ; 29)$ & $19(9 ; 31)$ \\
Nephropathy & $612(23)$ & $422(19)$ \\
Neuropathy & $843(32)$ & $578(26)$ \\
Retinopathy & $1,469(56)$ & $1,240(56)$ \\
Cardiovascular disease & $956(37)$ & $707(32)$ \\
Causes of death & & \\
Deaths during follow-up & $402(15)$ & $271(12)$ \\
Cardiovascular disease & $125(31)$ & $81(30)$ \\
Cancer & $51(13)$ & $45(17)$ \\
Acute complications of diabetes & $16(4)$ & $2(1)$ \\
Kidney disease & $8(2)$ & $10(4)$ \\
Accident & $12(3)$ & $9(3)$ \\
Lung disease (including pneumonia) & $15(4)$ & $17(6)$ \\
Infection & $31(8)$ & $23(9)$ \\
Gastrointestinal disease & $28(7)$ & $5(2)$ \\
Other & $116(29)$ & $79(29)$ \\
\hline
\end{tabular}

Values are median (interquartile range) or number of patients (\%) 

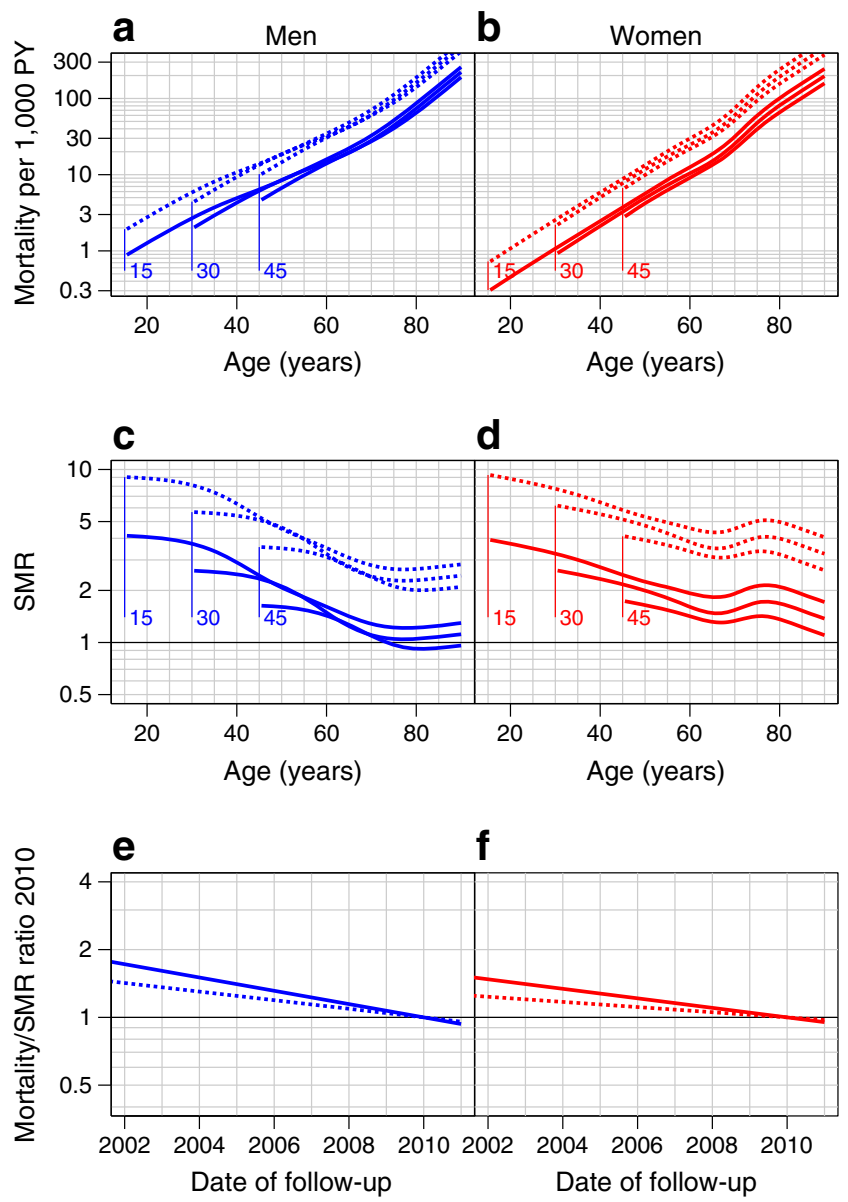

Fig. 1 (a, b) All-cause mortality/1,000 person-years (PY) for patients with (broken lines) and without (solid lines) nephropathy according to current age, sex and age at onset $(15,30$ and 45 years). Age-specific mortality rates are for 1 January 2010. (c, d) SMR for patients with (broken lines) and without (solid lines) nephropathy according to current age, sex and age at onset $(15,30$ and 45 years). Age-specific mortality rates are for 1 January 2010. (e, f) Mortality rate ratios (solid lines) and SMR ratios (broken lines) for all patients according to sex, with 1 January 2010 as the reference point

increased roughly exponentially with age (linearly on a log scale), and absolute mortality rates were higher in men than in women until the age of 65 years. Mortality rates were highest among those with the lowest age at onset, particularly among men. For example, mortality rates among men until the age of 60 were about $50 \%$ higher for onset of diabetes at age 15 compared with age 30 . A marked difference was found between patients with nephropathy and those without. Among men, mortality rates were 2.2 times higher, and among women 2.4 times higher, than in patients without nephropathy.

Standardised mortality rates Figure 1c, d shows SMR by age compared with the background population. SMR decreased with age for both men and women. Among men over 65, mortality rates were not significantly different from those in the background population. At younger ages, there was a strong inverse association between age at onset and mortality. Among women, the same trend was observed after the age of 60 , although not as strongly as among men; women diagnosed at age 45 had an old-age SMR of 1.3, whereas women diagnosed at age 15 had an old-age SMR of 2. For patients with nephropathy, SMR was higher in all age groups compared with patients without nephropathy.

Time trends in mortality rates Figure 1e, f shows mortality rate ratios with 1 January 2010 as the reference point. The time change in mortality rate was not significantly different for patients with and without nephropathy, and therefore values for all patients were combined. Mortality rates decreased linearly by calendar time: by $6.6 \%$ per year in men, by $4.8 \%$ per year in women (full lines). This was larger than the decrease in mortality rates in the background population. The decline in SMR was $4.3 \%$ and $2.6 \%$ per year among men and women (broken lines).

\section{Discussion}

The study evaluated absolute and relative mortality rates in patients with type 1 diabetes directly compared with the Danish background population. A significant finding is that the mortality rate among patients without late complications is only slightly elevated compared with the background population, emphasising the importance of aggressive early intervention to prevent the occurrence of complications. An encouraging finding is that mortality rates decreased in this period and more rapidly than in the background population. This study reveals that high mortality is still largely related to nephropathy.

Absolute mortality rates in the present study were similar to the results of age-comparable studies from the UK and USA $[4,7,8]$, but lower than found in a study from Switzerland [9]. Similar to other studies, the highest SMRs were found in the youngest age groups. However, while a young person diagnosed with type 1 diabetes has a very high SMR for all-cause mortality, their absolute risk is still relatively low because the background rate is low. Mortality among patients with nephropathy is somewhat lower than in other studies, which is probably due to the inclusion of microalbuminuria in the diagnostic criteria for nephropathy.

This study provides the most recent data on time trends in mortality rates with information available until the end of 2010. A few studies have reported a decrease in mortality rates in type 1 diabetes, particularly due to a decrease in late diabetic complications [8-10]. Some studies have reported no change, or an increase, in mortality rate $[1,11,12]$. This is partly a result of differences between populations and criteria for the definition of diabetes, but also a result of discrepancies 
in the duration of diabetes as well as differences in the time period between the studies.

Strengths and limitations of the study The strength of this study is the unselected population-based design, the precise information about diabetes type and complication status, and the precise estimates of mortality rates. Information on older patients with type 1 diabetes, including those diagnosed after the age of 30 , is particularly scarce in the literature. The main limitation is the relatively small sample of patients with type 1 diabetes. A second limitation is that the information on causes of death is based on death certificates rather than investigation of medical records. Third, the reference population includes the remaining estimated 20,000 patients with type 1 diabetes in Denmark; however, the small number of patients is unlikely to influence total mortality rates in the background population. The extent to which the remaining increased mortality risk is due to increased metabolic risk or lifestyle factors is not possible to determine because this information on these risk factors was not available.

In conclusion, the study reveals that (1) the absolute and relative mortality rates in patients with type 1 diabetes have decreased over the last 10 years, and more rapidly than in the background population, and (2) the high mortality rate in patients with type 1 diabetes is largely related to long-term diabetes complications.

Funding No external funding was received.

Duality of interest All three authors are employed by Steno Diabetes Centre A/S, a research hospital working in the Danish National Health Service and owned by Novo Nordisk A/S. All three authors own shares in Novo Nordisk A/S.

Contribution statement MEJ was responsible for study design, data management, and writing of the manuscript. $\mathrm{BC}$ was responsible for statistical analysis, interpretation of the results, and critical revision of the manuscript. TPA participated in interpretation of the results, and the writing and critical revision of the article. All authors had full access to all of the data in the study and fully approved the version to be published.

\section{References}

1. Patterson CC, Dahlquist GF, Harjutsalo VF et al (2007) Early mortality in EURODIAB population-based cohorts of type 1 diabetes diagnosed in childhood since 1989. Diabetologia 50:2439-2442

2. Borch-Johnsen KF, Andersen PK, Deckert T (1985) The effect of proteinuria on relative mortality in type 1 (insulin-dependent) diabetes mellitus. Diabetologia 28:590-596

3. Groop PH, Thomas MC, Moran JL et al (2009) The presence and severity of chronic kidney disease predicts all-cause mortality in type 1 diabetes. Diabetes 58:1651-1658

4. Livingstone SJ, Looker HC, Hothersall EJ et al (2012) Risk of cardiovascular disease and total mortality in adults with type 1 diabetes: Scottish Registry Linkage Study. PLoS Med 9:e1001321. doi:10.1371/journal.pmed.1001321

5. Dansk Voksen Diabetes Database. National årsrapport 2011, Version 1.4. Available from www.sundhed.dk/content/cms/88/4688_dvdd_ kommenteret_aarsrapport.pdf, accessed 25 July 2013

6. R Development Core Team (2013) R: A language and environment for statistical computing. R Foundation for Statistical Computing, Vienna

7. Soedamah-Muthu SS, Fuller JH, Mulnier HE, Raleigh VS, Lawrenson RA, Colhoun HM et al (2007) All-cause mortality rates in patients with type 1 diabetes mellitus compared with a nondiabetic population from the UK General Practice Research Database, 1992-1999. Diabetologia 49:660-666

8. Miller RG, Secrest AM, Sharma RK, Songer TJ, Orchard TJ (2012) Improvements in the life expectancy of type 1 diabetes: the Pittsburgh Epidemiology of Diabetes Complications Study cohort. Diabetes 61:1-6

9. Allemann S, Saner CF, Zwahlen MF, Christ ER, Diem PF, Stettler C (2009) Long-term cardiovascular and non-cardiovascular mortality in women and men with type 1 and type 2 diabetes mellitus: a 30-year follow-up in Switzerland. Swiss Med Wkly 139:576-583

10. Harjutsalo VF, Forsblom C, Groop PH (2011) Time trends in mortality in patients with type 1 diabetes: nationwide population based cohort study. BMJ 343:d5364

11. Waernbaum IF, Blohme GF, Ostman JF et al (2006) Excess mortality in incident cases of diabetes mellitus aged 15 to 34 years at diagnosis: a population-based study (DISS) in Sweden. Diabetologia 49:653-659

12. Burnet DL, Cooper AJ, Drum ML, Lipton RB (2007) Risk factors for mortality in a diverse cohort of patients with childhood-onset diabetes in Chicago. Diabetes Care 30:2559-2563 